\title{
THE ALVEOLAR BONE HEIGHT OF MANDIBULAR INCISORS AND MOLARS IN HEALTHY CHILDREN
}

\author{
Basheer Ali Mohammed Mabkhot*, Abeer Mostafa Abdellatif** and Ahmed Hamdy Ibrahim***
}

\begin{abstract}
Background: The most common periodontal disease in children is gingival inflammation around primary teeth, however, bone loss around the primary teeth has also seen and suggested as a sign of early periodontitis. Therefore it is important to detect the periodontal disease as early as possible in order to prevent the further destruction of tissues.
\end{abstract}

Aim: To determine the standard value of alveolar bone height.

Materials and Methods: 200 healthy children were joined this study. First group was 4-5 years of age and second group was 9-10 years of age. At least one side of the mandible was free of caries and gingival diseases. Digital radiographs were taken for the anterior and the posterior teeth of that side. Computer software (Planmeca Romexis Viewer) version 3.8.0. was used for drawing and measuring CEJ-ABC distance on radiograph.

Results: Group 1: The mean CEJ-ABC distance for the primary incisors was (1.06 $0.29 \mathrm{~mm})$ and for the primary molars was $(0.520 .13 \mathrm{~mm})$. Group 2: The mean CEJ-ABC distance for the permanent incisors was $(0.570 .09 \mathrm{~mm}$ and for the primary molars was $(0.800 .13 \mathrm{~mm})$. The mean CEJ-ABC distance for the first permanent molar was $\left(\begin{array}{lll}0.63 & 0.10 \mathrm{~mm}\end{array}\right)$. The increased CEJ-ABC distance of either of the incisors in-group 1 or the primary molars in-group 2 compared to their distance in the other age group is significant $(\mathrm{P} \leq 0.05)$.

Conclusion: This study provides valuable information on normal height of alveolar bone in two different age groups.

KEY WORDS: Alveolar bone height, Mandible, Cementoenamel Junction

\section{INTRODUCTION}

Periodontal disease is one of the most prevalent pathologies of the oral cavity, and it is found in all age groups ${ }^{(1-2)}$. There is a lot of important information about the diagnosis and treatment of periodontal diseases in adults, but there is relatively little information about the diagnosis, prevention and treatment of the periodontal diseases in children ${ }^{3}$.

* General Dentist, Faculty of Dentistry, Science and Technology University, Sana'a Yemen

** Professor of Pediatric Dentistry, Faculty of Dentistry Mansoura University

*** Assistant Professor of Pediatric Dentistry, Faculty of Dentistry Mansoura University 
The predominant form of periodontal disease in children and adolescents is gingivitis ${ }^{(1,4)}$. Gingivitis is the most important oral disease and the most prevalent among the pediatric population, exceeded only by dental caries ${ }^{(1,5-6)}$. Gingivitis of varying severity is almost universally present in the children and adolescents ${ }^{(2-3,6-7)}$. Periodontitis is less frequent during the childhood ${ }^{(3,5)}$.

Recent studies have indicated that juvenile periodontitisinthepermanentdentition of adolescents can often rapidly destroy the periodontium ${ }^{(8-9)}$. This indicates an importance of early recognition and the treatment of these patients to prevent the transition of the Early Onset Periodontitis (EOP), which is now considered as aggressive periodontitis, from the primary dentition to the permanent dentition. Children and adolescents susceptible to periodontal disease should be identified as early as possible in order to prevent the advance of a possibly destructive disease $^{10}$.

Studies have indicated that the periodontal disease in the permanent dentition of adolescents is often preceded by Bone Lose in the primary dentition ${ }^{9}$. Destruction of bone remains is the most important criterion for assessing the severity of periodontitis periodontal breakdown ${ }^{11}$. Bitewing radiographs are commonly taken in children for caries assessment and, in addition, these radiographs can also be used in order to observe the bone height around the first molars.

Thus, analyses of radiographs, used previously to caries analyzes, provide a good assessment of BL in children ${ }^{12-13}$. The radiographic signs as evidence of initial periodontal breakdown are (1) widening of the periodontal ligament space, (2) diffuseness or absence of the crest cortical plate, (3) thinning or absence of the trabeculae of the crestal alveolar, and (4) quantitative changes in the distance from the cementoenamel junction (CEJ) to the alveolar bone crest $(\mathrm{ABC}){ }^{14}$.

A previous literature review ${ }^{15}$ showed that the most objective criterion for the assessment of periodontal disease from radiographs is one that involves measuring the distance between the images of the CEJ and ABC. Furthermore, Pierro, et al. ${ }^{16}$ (2008) evaluated the reliability of methods caliper and computerized images to assess alveolar $\mathrm{BL}$ in primary teeth. Both methods were proven to be reliable. Nevertheless, there have been reports on the disadvantages of dental radiography as a diagnostic resource for detection of early periodontal lesions because it only reveals the interproximal aspects of the dentition ${ }^{15}$.

In this study, The Alveolar Bone Height of Mandibular Incisors and Molars in Healthy Children in a sample of Egyptian healthy children was assessed by analyzing the CEJ-ABC distance in bitewing and periapical radiographs collected at pediatric dentistry clinics in Mansoura university.

\section{MATERIALS AND METHOD}

This study was conducted on two age groups of children of both genders. The first group was preschool age children with an age ranging from 4-5 years. The second group was school age children with age ranging from 9-10 years. One hundred healthy children were included in each group.

Children who were visiting the pediatric dental clinic of faculty of dentistry of Mansoura University were screened to select suitable patients for this study. A written consent agreement was taken from the parents who accepted their children to participate in the study and approval of the ethical committee of the Faculty of Dentistry, Mansoura University was taken for this study.

Children included in this study were healthy and free from any systemic diseases. At least one side of the mandible was free of any deep proximal caries, interproximal restorations, calculus, heavy plaque and diastema.

Bitewing radiographs were taken for the primary molar area in the first group and for the primary and first permanent molar areas in the second group. Periapical radiographs were taken for the primary 
and permanent incisors areas in the first and the second groups. The dosage of $\mathrm{x}$-ray was $0.6 \mathrm{mSv}$ (millisievert).

On the radiographs three parallel horizontal lines were drawn subsequently; on the cusps of the tooth, cementoenamel junction (CEJ) and alveolar bone crest $(\mathrm{ABC})$. Another perpendicular line starts from the horizontal line of the tooth cusp to cross the parallel horizontal lines of the $\mathrm{CEJ}$ and $\mathrm{ABC}$, in which the parallel horizontal line was used as reference line for perpendicular line ${ }^{17}$. The distance between $\mathrm{CEJ}$ and $\mathrm{ABC}$ was measured by using Computer software (Planmeca Romexis Viewer) version 3.8.0.

A number of 10 radiographs were read by the examiner in two different occasions one-week apart. The results were statistically analyzed to determine the intra-examiner reliability. Another examiner (Hamdy A) was read the same 10 films and the re- sults were statistically analyzed to determine the inter-examiner reliability. Alpha Coronbaches factor

$$
\alpha \frac{N \bar{C}}{\bar{V}+(N-1) \bar{C}}
$$

was used to measure the intra-examiner and interexaminer reliability, where if the factor result is $60 \%$ or more, it considers acceptable. (Table 1)

All Means, standard deviations and descriptive statistics were analyzed by using (SPSS v. 22), the statistical package for the Social Sciences, to calculate the data of CEJ-ABC distance in mesial and distal surface in the two groups. Independent t-test was used for all comparison among groups, anterior and posterior teeth, and mesial and distal aspects, male and female too. ANOVA was used to analyze the difference between ages on the CEJ-ABC distances in the two groups.

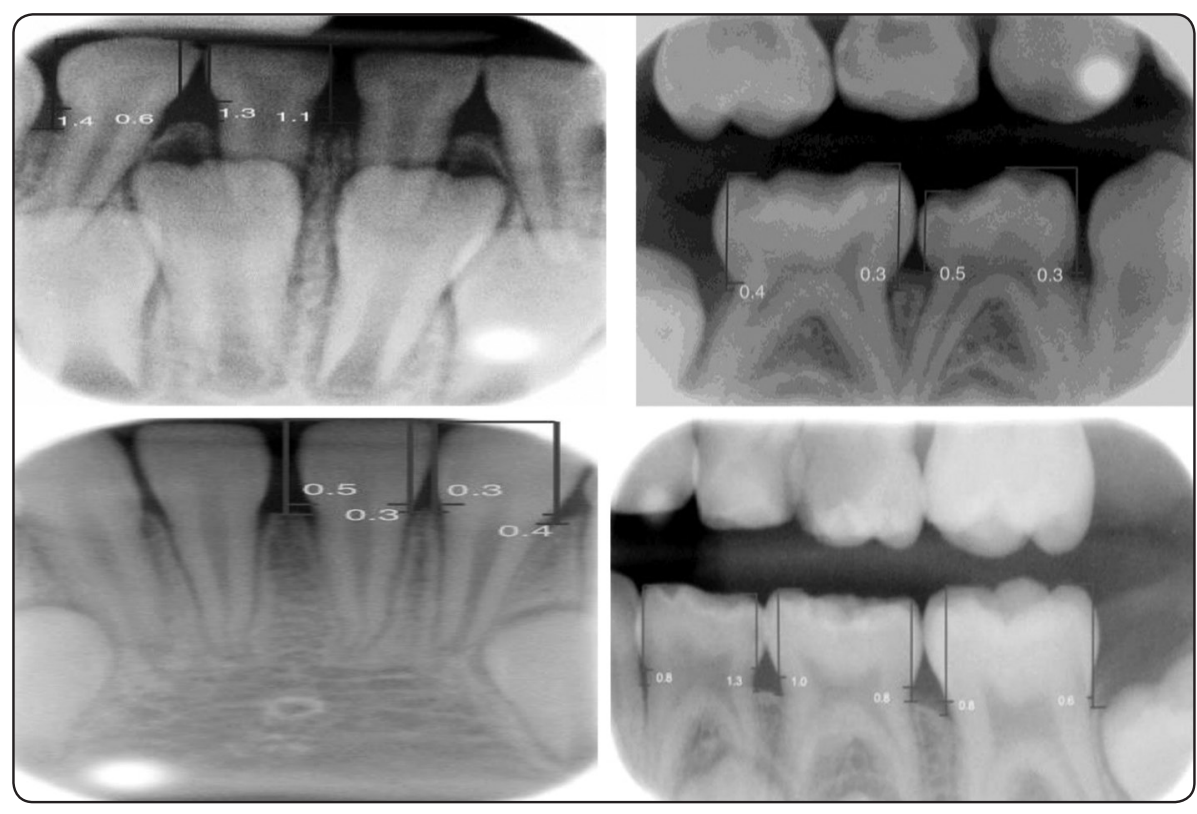

Fig. (1)

TABLE (1): Calibration and Reliability Measurements

\begin{tabular}{|c|c|c|c|c|c|c|c|c|}
\hline \multirow{2}{*}{ Teeth } & \multicolumn{2}{c|}{ A } & \multicolumn{2}{c|}{ B } & \multicolumn{2}{c|}{ D } & \multicolumn{2}{c|}{ E } \\
\hline Surfaces & M & D & M & D & M & D & M & D \\
\hline Alpha Coronbaches of Intraexaminer & $0.941 \%$ & $0.998 \%$ & $0.987 \%$ & $0.948 \%$ & $0.960 \%$ & $0.988 \%$ & $0.954 \%$ & $0.824 \%$ \\
\hline Alpha Coronbaches of Interexaminer & $0.992 \%$ & $0.924 \%$ & $0.948 \%$ & $0.985 \%$ & $0.947 \%$ & $0.741 \%$ & $0.652 \%$ & $0.828 \%$ \\
\hline
\end{tabular}




\section{RESULTS}

From 400 bitewing radiographs of 200 healthy girls and boys, totally, 1800 surfaces were measured and included in this study.

Table 2 displays the number of teeth, the mean, stander deviation, range, minimum and maximum distribution of CEJ-ABC distance in the anterior and posterior teeth of interproximal areas of primary incisors and molars in the first group. For the second group, table 3 displays the same values for permanent incisors, primary molars and first permanent molar.

The mean CEJ-ABC distance for the anterior and posterior teeth in the first group were 1.06 \pm 0.29 $\mathrm{mm}$ and $0.52 \pm 0.13 \mathrm{~mm}$, respectively). And (1.57 $\pm 0.09 \mathrm{~mm}$ and $0.80 \pm 0.13 \mathrm{~mm}$, respectively) for second group without first permanent molar. For the same group including the first permanent molar, the CEJ-ABC of posterior teeth was $(0.57 \pm 0.09 \mathrm{~mm}$ and $0.75 \pm 0.10 \mathrm{~mm}$, respectively) (table 4$). ? ?$

The general mean CEJ- ABC distance for all primary teeth in the first group was $(0.766 \pm 0.164$ $\mathrm{mm}$ ) and the general mean in the second group without the first permanent molar was $0.691 \pm$ 0.937 and with the first permanent molar was 0.680 \pm 0.848 (table 5).
The mean $\mathrm{CEJ}-\mathrm{ABC}$ distances for males and females in the first group, by applying the independent t-test, were found to be near ( and , respectively) and the difference was not statistically significant with $(\mathrm{P}=0.49)$. In the second group without presence of the first permanent molar was $(0.77 \pm 0.177 \mathrm{~mm}$ and $0.57 \pm 0.15 \mathrm{~mm}$, respectively) and the difference was not statistically significant $(\mathrm{P}=0.62)$. In the second group with presence of the first permanent molar was $(0.60 \pm 0.07 \mathrm{~mm}$ and $0.60 \pm 0.06 \mathrm{~mm}$, respectively) and the difference was not statistically significant $(P=0.70)($ table 6$)$.

The mean CEJ-ABC distance of the mesial and distal aspect in the first group was $(0.70 \pm 0.16 \mathrm{~mm}$ and $0.83 \pm 0.18$, respectively) the difference was statistically significant with $(\mathrm{P}=0.00)$. The mean CEJ-ABC distance of the mesial and distal aspect in the second group without the first permanent molar was (and, respectively) the difference was statistically significant with $(\mathrm{P}=0.00)$ as well as the mesial and distal aspect in the second group with the first permanent molar $(0.61 \pm 0.07 \mathrm{~mm}$ and $0.74 \pm 0.10 \mathrm{~mm}$, respectively) the difference was statistically significant with $(\mathrm{P}=0.00)($ table 8$)$

TABLE (2): The mean CEJ- ABC distance and distribution in the interproximal surfaces of the first group.

\begin{tabular}{|c|c|c|c|c|c|c|}
\hline & $\mathrm{N}$ & MEAN & SDT & RANGE & MINIMUM & MAXIMUM \\
\hline Aga & 100 & 4.520 & 0.502 & 1.00 & 4.00 & 5.00 \\
\hline MA & 100 & 1.368 & 0.336 & 1.40 & 0.60 & 2.00 \\
\hline DA & 100 & 1.013 & 0.431 & 1.80 & 0.20 & 2.00 \\
\hline MB & 100 & 0.637 & 0.356 & 1.50 & 0.00 & 1.50 \\
\hline DB & 100 & 1.252 & 0.380 & 1.60 & 0.40 & 2.00 \\
\hline MD & 100 & 0.352 & 0.132 & 0.80 & 0.00 & 0.80 \\
\hline DD & 100 & 0.652 & 0.204 & 1.50 & 0.10 & 1.60 \\
\hline ME & 100 & 0.443 & 0.178 & 0.90 & 0.00 & 0.90 \\
\hline DE & 100 & 0.412 & 0.120 & 0.60 & 0.20 & 0.80 \\
\hline
\end{tabular}

Abbreviation: Mesial (M) and Distal (D) reading for the primary teeth $(A, B, D, E)$ in-group 1. 
TABLE (3): The mean CEJ-ABC distance and distribution in the interproximal surfaces of second group.

\begin{tabular}{|c|c|c|c|c|c|c|}
\hline & $\mathrm{N}$ & MEAN & SDT & RANGE & MINIMUM & MAXIMUM \\
\hline Age & 100 & 9.440 & 0.498 & 1.00 & 9.00 & 10.00 \\
\hline M1 & 100 & 0.614 & 0.128 & 0.60 & 0.30 & 0.50 \\
\hline D1 & 100 & 0.589 & 0.152 & 0.70 & 0.30 & 1.00 \\
\hline M2 & 100 & 0.504 & 0.121 & 0.70 & 0.10 & 0.80 \\
\hline D2 & 100 & 0.588 & 0.135 & 0.50 & 0.30 & 0.80 \\
\hline MD & 100 & 0.602 & 0.092 & 0.50 & 0.50 & 1.00 \\
\hline DD & 100 & 1.026 & 0.219 & 1.00 & 0.50 & 1.50 \\
\hline ME & 100 & 0.766 & 0.190 & 1.00 & 0.40 & 1.40 \\
\hline DE & 100 & 0.842 & 0.192 & 0.90 & 0.50 & 1.40 \\
\hline M6 & 100 & 0.607 & 0.116 & 0.60 & 0.30 & 0.90 \\
\hline D6 & 100 & 0.662 & 0.119 & 0.60 & 0.30 & 0.90 \\
\hline
\end{tabular}

Abbreviation: Mesial (M) and Distal (D) reading for the permanent incisor $(1,2)$, primary molar $(D, E)$ and the first permanent molar (6) in group 2.

TABLE (4): comparison CEJ-ABC distance between anterior and posterior teeth among first and second groups

\begin{tabular}{|c|c|c|c|c|}
\hline & $\mathrm{N}$ & Mean \pm Std & t-test & p-value \\
\hline G1 anterior teeth & 100 & $1.067 \pm 0.292$ & \multirow{2}{*}{16.025} & \multirow{2}{*}{0.00} \\
\hline $\mathrm{G} 2$ anterior teeth & 100 & $0.573 \pm 0.096$ & & \\
\hline G1 posterior teeth & 100 & $0.524 \pm 0.136$ & \multirow{2}{*}{14.799} & \multirow{2}{*}{0.00} \\
\hline G2 posterior teeth (without the first permanent molar) & 100 & $0.809 \pm 0.135$ & & \\
\hline G1 posterior teeth & 100 & $0.524 \pm 0.136$ & \multirow{2}{*}{13.024} & \multirow{2}{*}{0.00} \\
\hline G2 posterior teeth (with the first permanent molar) & 100 & $0.751 \pm 0.107$ & & \\
\hline
\end{tabular}

Abbreviation: the first and second groups $(G 1, G 2)$

TABLE (5): Comparison of general mean of CEJ-ABC distance among first and second groups

\begin{tabular}{|l|c|c|c|c|}
\hline \multicolumn{1}{|c|}{ Group } & $\mathrm{N}$ & Mean \pm Std & t-test & p-value \\
\cline { 1 - 3 } G1 & 100 & $0.7661 \pm 0.16499$ & 3.939 & 0.00 \\
\cline { 1 - 3 } G2 (without the first permanent molar) & 100 & $06914 \pm 0.9377$ & \multirow{2}{*}{4.642} & 0.00 \\
\hline G1 & 100 & $0.7661 \pm 0.166499$ & & \multirow{2}{*}{$0.6800 \pm 08486$} \\
\hline
\end{tabular}


TABLE (6): comparison CEJ-ABC distance between male and female in first and second groups

\begin{tabular}{|l|c|c|c|c|}
\hline \multicolumn{1}{|c|}{ Group } & $\mathrm{N}$ & Mean \pm Std & t-test & p-value \\
\cline { 1 - 3 } G1 males & 100 & $0.776 \pm 0.177$ & \multirow{2}{*}{0.680} & 0.498 \\
\hline G1 females & 100 & $0.753 \pm 0.150$ & & \multirow{2}{*}{0.377} \\
\cline { 1 - 3 } G2 males & 100 & $0.608 \pm 0.073$ & $0.602 \pm 0.067$ & \\
\hline
\end{tabular}

TABLE (7): Comparison of CEJ-ABC distance between mesial and distal aspects within first and second group.

\begin{tabular}{|c|c|c|c|c|}
\hline Group & $\mathrm{N}$ & Mean \pm Std & t-test & p-value \\
\hline M-G1 & 100 & $0.700 \pm 0.169$ & \multirow{2}{*}{5.208} & \multirow{2}{*}{0.00} \\
\hline D-G1 & 100 & $0.832 \pm 0.188$ & & \\
\hline M-G2 (without the first permanent molar) & 100 & $06215 \pm 0.085$ & \multirow{2}{*}{9.512} & \multirow{2}{*}{0.00} \\
\hline D-G2 (without the first permanent molar) & 100 & $0.761 \pm 0.124$ & & \\
\hline M-G2 (with the first permanent molar) & 100 & $0.618 \pm 0.076$ & \multirow{2}{*}{9.390} & \multirow{2}{*}{0.00} \\
\hline D-G2 (with the first permanent molar) & 100 & $0.741 \pm 0.106$ & & \\
\hline
\end{tabular}

Abbreviation: Mesial aspect (M) and Distal aspect (D) reading for the first and second groups (G1, G2).

\section{DISCUSSION}

The aim of this study was to determine the height of alveolar bone of mandibular incisors and molars, and to establish the standard value of alveolar bone height in two age groups of healthy Egyptian children. Then data can be linked to different physiological and pathological factors in purpose to assess the early periodontal disease ${ }^{18}$. The comparison between the normal distance of CEJ$\mathrm{ABC}$ in 4-5years old and 9-10 years old of children may allow us to trace the change on this distance and could help us to detect the early periodontal disease in pediatric patient.

Periapical and bitewing radiographs (phosphor plates) were selected to measure the distance of CEJ-ABC as usual usage in first dental visit in pediatric patient for revealing the initial stages of development of interproximal decay even before they are emerged clinically and according to the American Academy of Pediatric Dentistry (AAPD Guidelines $)^{19}$. Also the bone height of the mandible is displayed on these radiographs, Many others studies use non digital radiographs films.

Traditional analogue radiographs cannot be changed once they are processed. The exposure conditions and the developing procedure determine the final result as well as that brightness and contrast are fixed. Digital radiographs, however, can be altered after they have been produced. This can be used to correct overexposure or underexposure of an image, and it can help to rescue an image in which exposure conditions were not optimal and thus prevent the need for a remake, saving the patient from an extra dose of radiation ${ }^{20}$.

Clinical examination of detecting the attachment loss in primary teeth was not taken in this study's 
consideration, since different studies have displayed that the migration of junctional epithelium apically into the root surface of primary tooth and this migration could be associated to continuous eruption ${ }^{18,21}$. In this study, all the examined cases were having no excessive apical root resorption as indicated by the radiographs because it was reported that apical root resorption more than $3 \mathrm{~mm}$ may disturb the height of alveolar bone crest ${ }^{18}$.

All children of this study were selected carefully according to requested criteria. This comes with disagreement with another studies where they choose their pediatric patient randomly ${ }^{12,22-23}$. This disagreement of the study sample select could be related to the aim of study.

In this work, one side, which was suitable for study, of the mandible was selected. This was in accordance to other studies that have displayed that no significant difference in the measurement between both sides of mouth ${ }^{21,24}$.

The smallest mean CEJ-ABC distance was registered at the mesial aspect of the first primary molar in first group $(0.35 \mathrm{~mm})$ and this finding was near with the results of other study where the smallest measure of CEJ-ABC was in mesial aspect of the first primary molar $(0.39 \mathrm{~mm})^{25}$. This smallest mean may due to the curving zone at angle of mandible that radiographic project in 9,26 or different anatomy of teeth and mandible or may be attributed to eruption of permanent teeth and exfoliation of the primary teeth ${ }^{13}$. In another study, the lowest mean of CEJ-ABC was in the mandibular second primary molar ${ }^{27}$.

The mean of CEJ- ABC distance of posterior teeth in the second group without the first permanent molar was $(0.80 \mathrm{~mm})$ and Maximum value did not exceed $(1.02 \mathrm{~mm})$, which was close to the result of another study for Bimstein et al ${ }^{28}$, where the mean of CEJ-ABC distance for the first and second primary molars were $0.88 \mathrm{~mm}$ as well as study for Jamal et al, the mean of CEJ-ABC distance for the first and second primary molars were $0.95 \mathrm{~mm}$ 25. The difference between these results of these studies reflect the different methods used in each study for example the usage of magnifying lenses, digital sliding gauges and computer-software measurements.

In this study, significant differences have been observed between mesial and distal surfaces, however, this findings were close with result of other study where the mesial surfaces had less value than distal surfaces ${ }^{25}$ as well as another study for Sardana V et al ${ }^{18}$. On the other hand, a study stated that there is no difference between mesial and distal surface in mean of CEJ-ABC distance 17. The variation between different studies may be due to that the CEJ of mesial aspect is not at same level of that distal aspect or technique of assessing radiographs or differences in sample size or may be the surfaces measured were not comparable in terms of dental decay, filling, and nearness of eruption or exfoliation time ${ }^{25}$.

In this study, there was no difference showed between males and females among groups and this finding was comparable with the results of other studies where males were not showing any differences in the mean of CEJ-ABC distance to females ${ }^{25,28,29-30}$. Conversely, another study showed that the males have higher measurement of CEJ$\mathrm{ABC}$ distance than those of females have ${ }^{17-18}$. This difference might be attributed to the randomization of sample selection in the other studies.

According to the age, this study showed no difference in the CEJ-ABC distance inside groups, but it exhibited different between the two groups. However, this is anticipated due to the exfoliation of primary teeth and eruption of permanent teeth happens between the two groups. In this study, the findings of first group (4 to 5 years old) is similar to other study where the results displayed that age had no effect on the distance CEJ-ABC in the primary teeth phase, between 2 and 5 years of age of Brazilian 
children only ${ }^{31}$. Also, the findings of second group (9 to 10 years old) were similar to other study where there was no difference among the four age groups (6-9 years) and its mean distances where becoming somewhat smaller with age, but there is no any relevance to this drop when measurements become beneath the threshold CEJ-ABC distance ${ }^{25}$.

\section{CONCLUSION}

This study supplies valuable information on normal height of alveolar bone in two different age groups of healthy Egyptian children. The CEJ-ABC distance difference between the two age groups is most likely related to the shedding process changes.

\section{REFERENCES}

1 - Chile. Ministerio de Salud. Regulation for the prevention of gingival and periodontal diseases. Human Health Division. Department of Odontology. Chilean Health Ministry; 1998.

2 - Feldens EG, Feldens CA, Kramer PF, Ferreira SH. Distribution of plaque and gingivitis and associated factors in 3- to 5-year-old Brazilian children. J Dent Child 2006;23:4-10.

3 - Escobar F. Pediatric Odontology. 2nd ed. Caracas (Venezuela): Actualidades Médico- Odontológicas Latinoamérica; 2004.

4 - Mariotti A. Dental plaque-induced gingival diseases. Ann Periodontol 1999;4:7-17.

5 - American Academy of Periodontology. Guideline on periodontal diseases in children and adolescents. J Periodontol 1996;67:57-62.

6 - Palma P, Gajardo M. Periodontal pathogens in children from 8 to 11 years old in two schools of the Metropolitan Region. Association with gingivitis. Rev Soc Chil Odontopediatría 2008;23:40.

7 - Pinkham JR. Odontología Pediátrica. 3rd ed. Mexico City: McGraw-Hill Interamericana Editores; 2001.

8 - Sjodin B, Crossner C.G, Unell, Ostland, P. A retrospective radiographic study of alveolar bone loss in the primary dentition in-patients with localized juvenile periodontitis. J Clin Periodontol 1989;16:124-127.
9 -Sjodin B, Mattsson L. Marginal bones loss in the primary dentition of patients with juvenile periodontitis. J Clin Periodontol. 1993; 20: 321- 36.

10 - Shariatmadar R. Torkzaban, P. Gholami, L. Cementoenamel junction-alveolar bone crest distance in interproximal areas of intact primary molars in healthy 7-9 year old girls in Hamadan. DJH 2010; Vol.2, No.1.

11 - Nowak JM. Classification of diseases and conditions affecting the periodontium. In: Newman MG, Takei HH, Carranza FA, eds. Clinical Periodontology. Chicago: WB Saunders; 2001.p.64-73.

12 - Bimstein E, Treasure ET, Williams SM, Dever JG. Alveolar bone loss in 5 years-old New Zealand children: its prevalence and relationship to caries prevalence, socioeconomic status and ethnic origin. J Clin Periodontol. 1994;21(7):447-50.

13 - Shapira L, Tarazi E, Rosen L, Bimstem E. The relationship between alveolar bone height and age in the primary dentition. A retrospective longitudinal radiographic study. J Clin Periodontol. 1995;22(5):408-12.

14 - Mann J, Pettigrew R, Beideman P, Green P, Ship I. Investigation of the relationship between clinically detected loss of attachment and radiographic changes in early periodontal disease. J Clin Periodontol. 1985;12(3):247-53.

15 - Keszthelyi G, Szabó I. Attachment loss in primary molars. J Clin Periodontol. 1987;14(1):48-51.

16 - Pierro VS, Souza IP, Luiz RR, Barcelos R, Moraes RS. Reliability of two methods for measurement of alveolar bone level in children. Dentomaxillofac Radiol. 2008;37(1):34-9.

17 - Needleman HL, Ku TC, Nelson L, Allred E, Seow WK. Alveolar bone height of primary and first permanent molars in healthy 7-t to 9-year- old children. ASDC J Dent child 1997:64(3): 188-196.

18 - Sardana v, Balappanavar A, Deshpande S, Shigli A, Indushekar KA, Gogia G. Evaluation of marginal alveolar bone height for early detection of periodontal disease in pediatric population: clinical and radiographic study. The journal of contemporary dental practice, 4014;15(1):37-45.

19 - Guideline on prescribing dental radiographs for infants, children, adolescents, and persons with special health care needs. Pediatr Dent 2008; 30 (7 Suppl): 236-237.

20 - Paul F. van der Stelt. Better imaging the advantages of digital radiography. Elsevier Inc. JADA 2008; 139(6 supplement): $7 \mathrm{~S}-13 \mathrm{~S}$. 
21 - Bimstein E, Soskolne A. W. A radiographic study of interproximal alveolar bone crests between the primary molars. ASDC J Dent for child 1984; 55:348-350.

22 - Da Silva PVS, De Souza IPR. Alvrolar bone loss in primary dentition. State of the Art. J Clin Pediatr Dent 2004; 29(2); 139-142.

23 - Bimstien E, Shapira L. Landau E, Sela MN. The relationship between alveolar bone loss and proximal caries in children; prevalence and microbiology. ASDC J Dent child 1993;60(20):99-103

24 - Bimstien E, Ranly DM, Skjonsby S, Soskolne WA. The effect of the facial growth, attrition, and age on the distance from the cementoenamel junction to the alveolar bone crest in the deciduous dentition, Am J orthod dentofacial orthop 1993;103(6);521-525.

25 - G. A. Jamal, O. Al-Batayneh \& D. Hamamy. The alveolar bone height of the primary and first permanent molars in healthy 6- to 9-year-old Jordanian children. International Journal of Paediatric Dentistry 2010: 151-159.
26- Kallestal C, Matsson L. Criteria for assessment of interproximal bone loss on bite-wing radiographs in adolescents. J Clin Periodontol 1989; 16: 300-304.

27 - Darby IB, Lu J, Calache H. Radiographic study of the prevalence of periodontal bone loss in Australian schoolaged children attending the Royal Dental Hospital of Melbourne. J Clin Periodontol 2005; 32: 959-965.

28 - Bimstein E, Delaney JE, Sweeney EA. Radiographic assessment of the alveolar bone in children and adolescents. Pediatr Dent 1988; 10: 199-204.

29 - Hart TC, Marazita ML, Schenkein HA, Brooks CN, Gunsolley JG, Diehl SR. No female preponderance in juvenile periodontitis after correction for ascertainment bias. J Periodontol 1991; 62: 745-749.

30 - Loe H, Brown LJ. Early onset periodontitis in the United States of America. J Periodontol 1991; 62: 608-616.

31 - Guimaraes MCM, Araujo VM, Avena MR, Duarte DRS, Freitas FV. Prevalence of Alveolar Bone Loss in Healthy Children Treated at Private Pediatric Dentistry Clinics. J Appl Oral Sci. 2010; 18 (3): 285-90 